\title{
Bone mineral density and fracture risk in leprosy affected people with leprosy reaction
}

\author{
Farhana Quyum $^{\mathrm{a}}$, Mashfiqul Hasan ${ }^{\mathrm{b}} \&$ Mohammad Atiqur Rahman ${ }^{\mathrm{b}}$ \\ a Associate Professor, Dermatology and Venereology, Ashiyan Medical \\ College Hospital, Dhaka, Bangladesh \\ ${ }^{\mathrm{b}}$ Assistant Professor of Endocrinology, National Institute of Neurosciences \\ and Hospital, Dhaka, Bangladesh
}

Submitted 27 July 2020; Accepted 10 August 2020

\begin{abstract}
Summary
Background: Bone health can be seriously affected by the leprosy reaction itself and steroids used in its treatment.

Objective: To assess the bone mineral density (BMD) by dual-energy X-ray absorptiometry (DXA) scan and fracture risk by the fracture risk assessment tool (FRAX) in steroid treated patients with leprosy reaction.

Methods: This study involved 25 patients with leprosy reaction treated with steroids (prednisolone) and 22 healthy family members attending the urban leprosy clinics of The Leprosy Mission International - Bangladesh (TLMI-B) Dhaka program in January-March, 2018. Socio-demographic and clinical information was recorded and BMD was measured in the spine, femur neck and forearm by DXA. Fracture risk was calculated by FRAX.

Results: Both the T-score and Z-score of the spine were lower in the leprosy group in relation to those of the control group [leprosy vs. control: T-score $-1.7(-3.2-1.3)$ vs. $-1.2(-2.5-0.8), p=0.030$ and Z-score $-1.9(-3.3$ to -1.4$)$ vs. $-1.2(-2.3$ to -0.2$)$, $p=0.038$; median (interquartile range). In other regions (femur neck, forearm) both scores were similar between the two groups, except for Z-score of the left femur neck which was lower in the leprosy group $(p=0.036)$. Although the frequency of osteopenia and osteoporosis in different sites was similar between groups, the 10-year probability of major osteoporotic fracture [leprosy vs. control: $2.2 \%(1.6-4.7)$ vs. $1.1 \%(0.9-2.0)$; $p<0.001$ ] or hip fracture [leprosy vs. control: $0.4 \%(0.1-1.7)$ vs. $0.1 \%(0.0-0.2) ; p$ $=0.001]$ by FRAX was higher in the leprosy group.

Conclusions: Participants with leprosy reactions and steroid treatment had lower BMD related parameters in lumber spine and higher risk of osteoporotic fracture.
\end{abstract}

Keywords: Leprosy, bone mineral density, fracture risk

Correspondence to: Dr. Farhana Quyum, Associate professor of Dermatology, Ashiyan Medical College Hospital, Dhaka, Bangladesh (Tel.: 01816268745; e-mail: farhana.quyum@gmail.com) 


\section{Introduction}

In many countries of the world including Bangladesh, the transmission of leprosy has been reduced and the 'elimination goal' has been achieved. ${ }^{1}$ At present the focus has shifted to the reduction of disability in residual patients with leprosy. In many patients who complete multi-drug therapy for leprosy, the morbidity continues in the form of lepra reaction requiring repeated steroid therapy. ${ }^{2}$ It may seriously affect the bone health and may lead to osteoporosis. ${ }^{3}$ The inflammatory mediators of lepra reactions negatively affect bone remodeling as well. ${ }^{4}$ Nutritional deficiencies and immobility further increase the risk of osteoporosis. In male patients, testicular involvement results in deficiency of testosterone. ${ }^{5}$ In addition to the negative impact on sexual health, this can perpetuate osteoporosis. ${ }^{6,7}$ In spite of having so many predilections, osteoporosis in steroid treated leprosy reaction patients has not been well explored.

Osteoporosis compromises bone strength and results in an increased risk of fracture. The method of choice for its diagnosis is measurement of bone mineral density (BMD) by dualenergy X-ray absorptiometry (DXA) expressed by the bone mineral content $\left(\mathrm{g} / \mathrm{cm}^{2}\right)$, T-score and Z-score measured in the lumber vertebrae, diaphysis of the radius and femur neck. ${ }^{8}$ The T-score denotes a comparison of the bone density of a particular person with that of healthy, young individuals of the same sex. It measures by how many standard deviations the patient's BMD value differs from that of a young healthy control. A negative T-score of -2.5 or below defines osteoporosis. T-scores between -1 and -2.5 indicate osteopenia and values above -1 are regarded as normal. On the other hand, Z-score is a comparison of the bone density of a particular person with that of healthy individuals of the same age and sex. It measures by how many standard deviations the BMD deviates from that of an age matched control. A low Z-score raises suspicion of a secondary cause of osteoporosis. ${ }^{8}$

However, in low-resource areas this test is not widely available or feasible as a screening test. Despite having a good specificity to predict fragility fracture, a major drawback of BMD assessment is its low sensitivity to predict the same. ${ }^{9}$ This is because of the fact that fracture risk is not solely dependent on BMD, while other clinical risk factors like smoking, alcohol consumption and use of corticosteroids are closely related to it. To address these limitations of BMD, the World Health Organization (WHO) introduced a fracture risk assessment tool (FRAX) which is a computer based algorithm that estimates the 10 -year probability of hip fracture and major osteoporotic fracture for untreated patients aged between 40 and 90 years by means of easily obtainable risk factors for fracture, with or without femoral neck BMD. ${ }^{10}$ Since its introduction, FRAX has been found to be a very useful assessment tool and it is now approved by the National Institute for Health and Care Excellence, UK and the Food and Drug Administration, USA. ${ }^{11}$ It is established as the standard global tool for fracture risk assessment and is used by a number of healthcare professionals internationally. ${ }^{12}$ It has not been used to screen fracture risk in leprosy affected people. With this background, we conducted an observational study to assess the BMD by DXA scan and fracture risk by FRAX in leprosy affected people with leprosy reaction who required steroid treatment.

\section{Materials and methods}

\section{STUDY SUBJECTS}

In this observational study, 25 leprosy affected people with leprosy reaction treated with prednisolone within last 6 months were enrolled from the urban leprosy clinics of The Leprosy Mission International - Bangladesh (TLMI-B) Dhaka program, from January to March 2018. 
Twenty-two of their family members, who had no clinical features of leprosy or any other major illness, were enrolled as controls. None of the participants had had their bone mineral density checked or were taking any treatment for osteopenia/osteoporosis before the study. Socio-demographic and clinical information was recorded in a semi-structured questionnaire; height, weight and blood pressure were recorded. Duration of steroid therapy was assessed by history and from medical records.

\section{STUDY PROCEDURE}

After recruitment, the participants were instructed to come for measurement of BMD by DXA along with a healthy family member of similar age. BMD was measured in the lumber spine, both femur necks and the left forearm. A Lunar Prodigy Advance bone densitometer (GE Healthcare, Madison, WI, USA) was used.

Fracture risk was calculated with the FRAX tool which predicts the 10-year probability of major osteoporotic fracture or hip fracture using clinical risk factors, with or without BMD. The clinical risk factors included in the FRAX algorithm are: age, sex, weight, height, previous fracture, parental hip fracture, current smoking, use of glucocorticoids, presence of rheumatoid arthritis or secondary osteoporosis and alcohol intake. FRAX is also calibrated according to fracture probability for different regions of the world, where the epidemiology of fracture is known. As 'Bangladeshi' ethnicity is not included in the FRAX online calculator, we used 'Sri Lanka' as they have a close epidemiological resemblance to our population. For participants younger than 40 years, we used their actual age as input, although the software calculates the fracture risk assuming their age as 40 .

\section{ETHICAL ASPECTS}

The study was performed with the approval of the Ethical Review Committee of the Ashiyan Medical College Hospital, Dhaka. Written informed consent was taken from the participants.

\section{STATISTICAL ANALYSIS}

Data were analyzed using IBM SPSS Statistics for Windows version 22.0 (IBM Corp, Armonk, NY, USA). Quantitative data were expressed as mean and standard deviation, if normally distributed, while median values with interquartile ranges (IQR) were used, if not normally distributed. Qualitative data was expressed as frequency and percentage. For continuous variables, comparison between groups was made by the Student's $t$-test or Mann-Whitney $U$ test. Categorical variables were analyzed by the $\chi^{2}$ test. Statistical significance was accepted at $p=0.05$.

\section{RESULTS}

The study recruited 25 leprosy affected people, and 22 healthy family members as controls (Table 1). Although there was no significant difference between these two groups in relation to age, monthly income of the family, tobacco use, height, weight, body mass index (BMI) and blood pressure), there was a male predominance in leprosy group and a female predominance in control group $(p=0.002)$. The frequency of sedentary lifestyle was also higher in participants within the leprosy group $(p=0.031)$. All the participants with leprosy had a history of reactions (mostly type-II, 60\%) (Table 2). Nearly half of them had grade-II disability (44\%) and most $(76 \%)$ had completed their MDT before the study. All patients had a history of treatment with prednisolone , having been treated for $<3$ months (36\%), 3-12 months (36\%) or $>12$ months 
Table 1. Characteristics of the study participants

\begin{tabular}{|c|c|c|c|c|}
\hline Characters & $\begin{array}{l}\text { Leprosy group } \\
\quad(n=25)\end{array}$ & $\begin{array}{l}\text { Control group } \\
\quad(n=22)\end{array}$ & $\begin{array}{l}\text { All participants } \\
\qquad(n=47)\end{array}$ & ${ }^{*} p$-value \\
\hline Age $($ mean $\pm S D)$ & $40.4 \pm 13.8$ & $37.6 \pm 12.6$ & $39.1 \pm 13.2$ & 0.479 \\
\hline $\begin{array}{l}\text { Gender } \\
\text { Male } \\
\text { Female }\end{array}$ & $\begin{array}{c}19(76.0) \\
6(24.0)\end{array}$ & $\begin{array}{c}7(31.8) \\
15(68.2)\end{array}$ & $\begin{array}{l}26(55.3) \\
21(44.7)\end{array}$ & 0.002 \\
\hline $\begin{array}{l}\text { Occupation category } \\
\text { Sedentary } \\
\text { Not-sedentary }\end{array}$ & $\begin{array}{c}18(72.0) \\
7(28.0)\end{array}$ & $\begin{array}{c}9(40.9) \\
13(59.1)\end{array}$ & $\begin{array}{l}27(57.4) \\
20(42.6)\end{array}$ & 0.031 \\
\hline $\begin{array}{l}\text { Monthly income } \\
\leq 10,000 \\
>10,000\end{array}$ & $\begin{array}{l}14(56.0) \\
11(44.0)\end{array}$ & $\begin{array}{c}15(68.2) \\
7(31.8)\end{array}$ & $\begin{array}{l}29(61.7) \\
18(38.3)\end{array}$ & 0.391 \\
\hline $\begin{array}{l}\text { Tobacco user } \\
\text { Height (in } \mathrm{cm}) \\
\text { Weight (in kg) } \\
\text { BMI (in } \mathrm{kg} / \mathrm{m} 2) \\
\text { Systolic BP (mm Hg) } \\
\text { Diastolic BP (mm Hg) }\end{array}$ & $\begin{array}{c}18(72.0) \\
157.2 \pm 7.3 \\
54.9 \pm 7.5 \\
22.3 \pm 3.3 \\
118.8 \pm 22.7 \\
77.6 \pm 17.1\end{array}$ & $\begin{array}{c}11(50.0) \\
152.6 \pm 9.9 \\
52.9 \pm 8.7 \\
22.8 \pm 3.9 \\
115.9 \pm 20.9 \\
76.4 \pm 10.9\end{array}$ & $\begin{array}{c}29(61.7) \\
155.1 \pm 8.8 \\
54.0 \pm 8.1 \\
22.6 \pm 3.6 \\
117.7 \pm 21.6 \\
77.0 \pm 14.4\end{array}$ & $\begin{array}{l}0.122 \\
0.077 \\
0.399 \\
0.612 \\
0.653 \\
0.773\end{array}$ \\
\hline
\end{tabular}

${ }^{*}$ Significance level stands for difference between leprosy group and control group by independent $t$-test or $\chi^{2}$ test, as applicable. Within parentheses are percentages over column total.

$(28 \%)$. A majority of patients $(84 \%)$ had had the last dose of prednisolone less than 3 months before enrollment.

The frequency of osteopenia and osteoporosis at different sites was similar between the groups (Table 3). The frequency of a BMD indicating osteoporosis was higher for the spine in both the leprosy group and the control group. Both the T-score and Z-score for the spine were significantly lower in the leprosy group, compared to the control group (Table 4). But the T-score or Z-score for other regions (femur neck, forearm) were comparable between the two groups, except for Z-score of the left femur neck, which was significantly lower in the leprosy group ( $p=0.036)$. The 10-year probability of a major osteoporotic fracture for leprosy vs. control (median and IQR) was $2.2 \%(1.6-4.7)$ vs. $1.1 \%(0.9-2.0),(p<0.001)$ or hip fracture for leprosy vs. control (median and IQR) was $0.4 \%(0.1-1.7)$ vs. $0.1 \%(0.0-0.2),(p=0.001)$, as calculated by FRAX .

\section{DISCUSSIONS}

In this observational pilot study, BMD and fracture risk of leprosy affected people with a history of reactions requiring steroid therapy, were compared with unaffected family members. In the leprosy group, the T-scores and Z-scores of the lumbar spine were significantly lower in the leprosy group, while the risk of major osteoporotic fracture was 2-fold, and hip fracture 4fold, higher in comparison to their healthy peers. The frequency of osteopenia and osteoporosis was not statistically different between the groups. Osteoporosis and fracture risk are not well evaluated in leprosy patients across the world. However, a few previous studies have observed similar findings in leprosy patients. ${ }^{13,14}$

Around $50 \%$ of patients with leprosy may develop leprosy reactions prior to, during or after multi-drug therapy. ${ }^{15}$ These reactions are characterized by sudden reactivation of immune responses with high levels of cytokines such as IL-1, IL-6, IL-12, IFN- $\gamma$ and TNF. ${ }^{16}$ Although 
Table 2. Characteristics of the study participants with leprosy $(n=25)$

\begin{tabular}{lr}
\hline Character & Frequency $(\%)$ \\
\hline Type of reaction & $5(20.0)$ \\
Neuritis & $5(20.0)$ \\
Type-I & $15(60.0)$ \\
Type-II & \\
Disability & $11(44.0)$ \\
None & $3(12.0)$ \\
Grade-I & $11(44.0)$ \\
Grade-II & \\
MDT completion & $6(24.0)$ \\
On-going & $19(76.0)$ \\
Completed & \\
Duration of steroid therapy & $9(36.0)$ \\
3 months or less & $9(36.0)$ \\
$3-12$ months & $7(28.0)$ \\
$>12$ months & \\
Time since last dose of steroid & $21(84.0)$ \\
$0-3$ months & $4(16.0)$ \\
$3-6$ months &
\end{tabular}

Within parentheses are percentages over column total.

Table 3. Frequency of osteopenia and osteoporosis among the study participants $(n=47)$

\begin{tabular}{|c|c|c|c|c|}
\hline Character & $\begin{array}{l}\text { Leprosy group } \\
\quad(n=25)\end{array}$ & $\begin{array}{l}\text { Control group } \\
\quad(n=22)\end{array}$ & $\begin{array}{l}\text { All participants } \\
\quad(n=47)\end{array}$ & ${ }^{*} p$-value \\
\hline \multicolumn{5}{|l|}{ BMD of spine } \\
\hline Normal & $5(20.0)$ & $10(45.5)$ & $15(31.9)$ & \multirow{3}{*}{0.151} \\
\hline Osteopenia & $12(48.0)$ & $6(27.3)$ & $18(38.3)$ & \\
\hline Osteoporosis & $8(32.0)$ & $6(27.3)$ & $14(29.8)$ & \\
\hline \multicolumn{5}{|c|}{ BMD of femur neck (left) } \\
\hline Normal & $10(40.0)$ & $14(63.6)$ & $24(51.1)$ & \multirow{3}{*}{0.204} \\
\hline Osteopenia & $11(44.0)$ & $7(31.8)$ & $18(38.3)$ & \\
\hline Osteoporosis & $4(16.0)$ & $1(4.5)$ & $5(10.6)$ & \\
\hline \multicolumn{5}{|c|}{ BMD of femur neck (right) } \\
\hline Normal & $10(40.0)$ & $13(59.1)$ & $23(48.9)$ & \multirow{3}{*}{0.398} \\
\hline Osteopenia & $14(56.0)$ & $8(36.4)$ & $22(46.8)$ & \\
\hline Osteoporosis & $1(4.0)$ & $1(4.5)$ & $2(4.3)$ & \\
\hline \multicolumn{5}{|c|}{ BMD of forearm } \\
\hline Normal & $15(60.0)$ & $11(50.0)$ & $26(55.3)$ & \multirow{3}{*}{0.645} \\
\hline Osteopenia & $7(28.0)$ & $9(40.9)$ & $16(34.0)$ & \\
\hline Osteoporosis & $3(12.0)$ & $2(9.1)$ & $5(10.6)$ & \\
\hline
\end{tabular}

${ }^{*} \mathrm{By} \chi^{2}$ test. Within parentheses are percentages over column total. 
Table 4. Comparison of T-scores, Z-scores and fracture risk between leprosy group and control group

\begin{tabular}{lccc}
\hline Character & $\begin{array}{c}\text { Leprosy group }(n=25) \\
\text { (Median and IQR) }\end{array}$ & $\begin{array}{c}\text { Control group }(n=22) \\
\text { (Median and IQR) }\end{array}$ & ${ }^{*}$-value \\
\hline T-score of spine & $-1.7(-3.2$ to -1.3$)$ & $-1.2(-2.5$ to -0.8$)$ & $\mathbf{0 . 0 3 0}$ \\
Z-score of spine & $-1.9(-3.3$ to -1.4$)$ & $-1.2(-2.3$ to -0.2$)$ & $\mathbf{0 . 0 3 8}$ \\
T-score of femur neck (left) & $-1.3(-2.2$ to -0.8$)$ & $-0.9(-1.5$ to -0.5$)$ & 0.066 \\
Z-score of femur neck (left) & $-1.2(-1.8$ to -0.8$)$ & $-0.7(-1.4$ to -0.2$)$ & $\mathbf{0 . 0 3 6}$ \\
T-score of femur neck (right) & $-1.3(-1.9$ to -0.7$)$ & $-1.0(-1.2$ to -0.5$)$ & 0.124 \\
Z-score of femur neck (right) & $-1.1(-1.6$ to -0.6$)$ & $-0.7(-1.1$ to -0.2$)$ & 0.051 \\
T-score of forearm & $-0.9(-1.3$ to -0.3$)$ & $-1.1(-1.8$ to -0.4$)$ & 0.201 \\
Z-score of forearm & $-1.7(-2.7$ to -0.6$)$ & $-1.2(-2.0$ to -0.7$)$ & 0.481 \\
10-year probability of major & $2.2 \%(1.6-4.7)$ & $1.1 \%(0.9-2.0)$ & $<0.001$ \\
osteoporotic fracture & $0.4 \%(0.1-1.7)$ & $0.1 \%(0.0-0.2)$ & 0.001 \\
10-year probability of hip fracture & & & \\
\hline
\end{tabular}

*Significance level by Mann-Whitney $U$ test.

a number of drugs are observed to be effective, corticosteroids are still the mainstay of treatment for leprosy reactions. ${ }^{17}$ Bone loss is a serious side effect of this therapy which is mediated by direct inhibition of bone formation and increase in bone resorption. ${ }^{18}$ As a result, fracture risk increases; which is even higher in those requiring repeated or prolonged courses of steroid treatment. ${ }^{19}$ Despite these facts many patients treated with corticosteroids are not well evaluated for their skeletal health. Patients on glucocorticoids need to ensure adequate intake of vitamin D and calcium. Lifestyle modifications including regular exercise and elimination of other risk factors such as smoking or alcohol abuse is also recommended. The need for additional treatment is based on the risk stratification for which FRAX is helpful. There is a plethora of evidence to support pharmacologic treatment in both men and women at high risk of glucocorticoid induced osteoporosis. The first line agent has been, and remains, bisphosphonate therapy. If bisphosphonates are contraindicated, teriparatide is a suggested second line agent, followed by denosumab. ${ }^{20}$

In a study carried out in Istanbul, 87 leprosy patients were evaluated with DXA and compared to their healthy peers. ${ }^{13}$ Osteoporotic changes were observed at a higher rate in the femoral and lumber region of leprosy patients. Another study in Japan measured the BMD of 353 leprosy patients along with measurement of free testosterone in 81 male participants. ${ }^{14} \mathrm{~A}$ high frequency of osteoporosis in the participants was observed and their findings highlighted the importance of free testosterone in determining BMD in this group of patients. Low testosterone levels were also observed in Bangladeshi male leprosy patients in a separate study. ${ }^{5}$ This may be one of the key elements in the pathogenesis of osteoporosis in male leprosy patients.

The prevalence of osteoporosis in Bangladesh is not well studied. However, the frequency of osteoporosis in healthy family members of leprosy affected people may reflect that of general population of the country. The spinal BMD was found to be particularly affected in both groups, but more in the leprosy group. As effects of steroid therapy are more pronounced in the axial skeleton, the spine is particularly vulnerable.${ }^{18}$ Both the T-scores and Z-scores of the lumber spine are low in the leprosy group, indicating injury to the bone in addition to the agerelated decline in BMD. ${ }^{3}$ Pharmacological therapy for low BMD is indicated for those having a T-score of $\leq-2.5$. In the case of patients who have had steroid therapy, pharmacological therapy is indicated for a BMD at the level of 'osteopenia' (i.e. T-score between -1 to -2.5 ) to 
prevent fragility fracture. ${ }^{3}$ So, the number of persons who may benefit from pharmacological therapy is much higher in the leprosy group in comparison to the control group.

For prediction of osteoporosis-related fracture risk, clinicians in low-resource countries have found FRAX to be very suitable and cost-effective. ${ }^{21}$ In the absence of facilities for BMD estimation, FRAX can easily be used to estimate fracture risk and to identify people who need specific therapy for the prevention of fractures. We observed a 4-fold and 2-fold higher risk of hip fracture and major osteoporotic fracture respectively in people with leprosy reactions and corticosteroid therapy. Although the absolute risk of fracture was not very high in individual patients, it seems to be crucial to identify those with higher risk and to provide appropriate non-pharmacological and pharmacological treatment.

As a pilot study, our sample size was small, so we could not subdivide our study population according to age, gender or steroid dose-duration. It is well known that women start losing bone at an earlier age and at a faster rate than men. On the other hand, men usually have a fracture at a higher bone density. ${ }^{22}$ Sedentary behavior also negatively affects bone health. ${ }^{23}$ There was disparity in relation to gender and occupation distribution between the leprosy group and the control group in the present study, which may have affected the study findings. Bone health may also be different in the leprosy population under different economic conditions, different levels of disability and different degrees of testicular involvement affecting the testosterone level in men. To evaluate all these parameters, a comprehensive prospective study should be carried out.

In conclusion, leprosy affected people with a history of reactions and steroid treatment were observed to have lower BMD-related parameters (T-score and Z-score), particularly in the spine, and a higher risk of major osteoporotic fracture or hip fracture in relation to their unaffected peers.

\section{Acknowledgments}

The study was funded by TLMI-B (as part of an Advocacy for Empowerment Project - AEP component). We are grateful to all the staff of TLMI-B for their co-operation during the study.

\section{Conflicts of interest}

None declared.

\section{Contributorship}

Farhana-Quyum: Planning, implementation and manuscript writing Mashfiqul-Hasan: Planning, implementation and manuscript writing (Guarantor) M Atiqur-Rahman: Implementation and manuscript writing.

\section{References}

1 Ministry of health and family welfare. In: National Guidelines and Technical Manual on Leprosy. 3rd edn, Government of Bangladesh and World Health Organization, 2005; pp. 4-55.

2 Quyum F, Hasan M, Chowdhury WK, Wahab MA. Leprosy reactions: frequency and risk factors. $J$ Clin Dermatol Theor, 2016; 3: 022.

3 Fraser LA, Adachi JD. Glucocorticoid-induced osteoporosis: treatment update and review. Therapeutic Advances in Musculoskeletal Disease, 2009; 1(2): 71-85.

4 Hardy R, Cooper MS. Bone loss in inflammatory disorders. Journal of Endocrinology, 2009; 201(3): 309-320.

5 Hasan M, Quyum F, Atiqur-Rahman M, Sandesh-Panthi . Testicular dysfunction in men affected by lepromatous leprosy. Lepr Rev, 2017; 88: 258-264. 
6 Ishikawa S, Tanaka H, Mizushima M, Hashizume H, Ishida Y, Inoue H. Osteoporosis due to testicular atrophy in male leprosy patients. Acta Med Okayama, 1997; 51(5): 279-283.

7 Ishikawa S, Tanaka H, Mizushima M, Hashizume H, Ishida Y, Inoue H. Osteoporosis due to testicular atrophy in male leprosy patients. Acta Medica Okayama, 1997; 51(5): 279-283.

8 Sheu A, Diamond T. Diagnostic tests: bone mineral density: testing for osteoporosis. Australian Prescriber, 2016; 39(2): 35 .

9 World Health Organization. Assessment of fracture risk and its application to screening for postmenopausal osteoporosis. Report of a WHO study group. Geneva: World Health Organization; 1994. WHO Technical Report Series, 843.

10 FRAX $^{\circledR}$ WHO fracture risk assessment tool. Available from URL: https://www.sheffield.ac.uk/FRAX/ index.aspx (accessed 21 Mar 2020).

11 National Institute for Health and Care Excellence. NICE clinical guideline 146. Osteoporosis: assessing the risk of fragility fracture. London, UK: 2012.

12 Kanis JA, Johansson H, Harvey NC, McCloskey EV. A brief history of FRAX. Archives of Osteoporosis, 2018; 13(1): 118.

13 Bicer M, Ozucelık DN, Kultur A, Ozucelık SA, Kavak A, Cukurova Z. Evaluation of the relationship between osteoporosis and musculoskeletal deformities in leprosy patients through the DXA procedure. Int J Clin Exp Med, 2016; 9(3): 6621-6627.

14 Ishikawa S, Ishikawa A, Yoh K, Tanaka H, Fujiwara M. Osteoporosis in male and female leprosy patients. Calcified Tissue International, 1999; 64(2): 144-147.

15 Britton WJ, Lockwood DN. Leprosy. Lancet, 2004; 363(9416): 1209-1219.

16 Cortela DC, Nogueira MR, Pereira AC, Souza Junior AL, Ignotti E. Inflammatory cytokines in leprosy reactions and periodontal diseases. Revista do Instituto de Medicina Tropical de São Paulo, 2018; 60.

17 Martinez JD, Cardenas JA. Treatment of leprosy and leprosy reactions. Current Treatment Options in Infectious Diseases, 2017; 9(3): 287-298.

18 Canalis E, Mazziotti G, Giustina A, Bilezikian JP. Glucocorticoid-induced osteoporosis: pathophysiology and therapy. Osteoporos Int, 2007; 18(10): 1319-1328, doi:10.1007/s00198-007-0394-0.

19 Steinbuch M, Youket TE, Cohen S. Oral glucocorticoid use is associated with an increased risk of fracture. Osteoporosis International, 2004; 15(4): 323-328.

20 Hsu E, Nanes M. Advances in treatment of glucocorticoid-induced osteoporosis. Current Opinion in Endocrinology, Diabetes, and Obesity, 2017; 24(6): 411.

21 Akter N, Qureshi NK, Latif ZA. Can FRAX tool be used for determination of risk score for osteoporosis fractures in a financially constrained society like Bangladesh? BIRDEM Medical Journal, 2018; 8(1): 9-15.

22 Alswat KA. Gender disparities in osteoporosis. Journal of Clinical Medicine Research, 2017; 9(5): 382.

23 Chastin SF, Mandrichenko O, Helbostadt JL, Skelton DA. Associations between objectively-measured sedentary behaviour and physical activity with bone mineral density in adults and older adults, the NHANES study. Bone, 2014; 64: 254-262. 